\title{
How much of skin improvement over time in systemic sclerosis is due to normal ageing? A prospective study with shear-wave elastography.
}

Tania Santiago ( $\square$ tlousasantiago@hotmail.com )

Centro Hospitalar e Universitário de Coimbra https://orcid.org/0000-0002-1562-4022

Tania Louza Santiago

Centro Hospitalar e Universitario de Coimbra EPE

Mariana Galante Santiago

Centro Hospitalar e Universitário de Coimbra

Margarida Macedo Coutinho

Centro Hospitalar e Universitario de Coimbra EPE

Maria Joao Salvador

Centro Hospitalar e Universitário de Coimbra

José António Pereira Da Silva

Centro Hospitalar e Universitario de Coimbra EPE

Research article

Keywords: Skin, Stiffness, Shear-wave elastography, Systemic sclerosis, Ultrasound

Posted Date: February 18th, 2020

DOI: https://doi.org/10.21203/rs.2.18735/v2

License: (9) (i) This work is licensed under a Creative Commons Attribution 4.0 International License. Read Full License

Version of Record: A version of this preprint was published at Arthritis Research \& Therapy on March 18th, 2020. See the published version at https://doi.org/10.1186/s13075-020-02150-x. 


\section{Abstract}

Objective To assess changes in skin stiffness in SSc patients using SWE during a five-year follow-up. Methods Skin stiffness [i.e. shear-wave velocity values (SWV) in $\mathrm{m} / \mathrm{s}$ ] was assessed by SWE ultrasound (using virtual touch image quantification) at the 17 sites of the mRSS, in each participant, at baseline and follow-up. mRSS was performed at both time points. Differences between groups were analysed using the related-samples Wilcoxon Signed Rank test and the Mann-Whitney U test. Results We included 21 patients [85.7\% females; mean age 56.3 (10.4) years at baseline, $57.1 \%$ with limited SSc] and, 15 healthy controls [73.3\% females; mean age 53.6 (14.1) years)]. The median follow-up was $4.9(0.4)$ years. Skin stiffness decreased significantly at all Rodnan sites ( $p \leq 0.001)$, (except in the fingers), in SSc patients, over time. The same phenomenon occurred in controls, but to a lesser degree, in terms of percentage change. The percentage reduction in skin stiffness varied in the different Rodnan sites and in different phases of the disease. In addition, SWV values also decreased significantly in 15/16 skin sites with local normal Rodnan at baseline, whereas local Rodnan skin score only changed significantly in the upperarm $(p=0.046)$ and forearm $(p=0.026)$. Conclusion This study provides first-time evidence suggesting that skin SWV values are more sensitive to change over time than mRSS, and reduce significantly over time in SSc and normal controls.

\section{Introduction}

Skin involvement is a major feature of systemic sclerosis (SSc). ${ }^{1}$ The extent and rate of progression of skin fibrosis is of paramount importance as it correlates with functional limitations, internal organ involvement and survival. ${ }^{1}$ Therefore, measurement of skin involvement is not only essential for the diagnosis and assessment of prognosis in SSc, but also crucial to support the development of new therapies. The modified Rodnan skin score (mRSS), a semi-quantitative method based on palpation, is currently the gold standard measure of skin changes in SSc and is often the primary or secondary outcome measure in clinical trials. However, it has been criticised for its lack of objectivity, poor interobserver reproducibility and lack of sensitivity to change in skin thickness over time. ${ }^{2,3}$

Different ultrasound methods are being investigated as means to improved the assessment of skin involvement in SSc. High-frequency ultrasound offers a potential for objective, sensitive and reliable assessment of dermal thickness in SSc. ${ }^{4-6}$ However, it does not assess the tissue elastic properties.

In recent years, shear-wave elastography (SWE) has been investigated as a quantitative and operatorindependent tool to evaluate skin stiffness. ${ }^{7-9}$ Shear-wave velocity (SWV) values reflect tissue stiffness: the stiffer the tissue, the faster the shear-waves propagate. SWE may, therefore, provide a novel opportunity to objectively assess fibrosis - a crucial feature in the complex process of skin involvement in SSc. $^{10,11}$

Cross sectional studies have shown that SWV values are significantly higher in SSc patients than in controls, in almost all of the Rodnan sites. ${ }^{7-9}$ Interestingly, clinically unaffected skin of patients with SSc 
could also be differentiated from the skin of healthy comparators. ${ }^{7,8}$ Two previous studies have shown excellent reproducibility for SWV measurements, with inter-rater intraclass correlation coefficients (ICCs) ranging from 0.48 (phalanx) to 0.91 (upper arm). The corresponding values for intra-rater comparisons were 0.48 (chest) to 0.98 (phalanx). ${ }^{7,8}$

This is the first study to evaluate the progression of skin stiffness over-time with SWE in patients with SSc and in normal controls.

\section{Methods}

\section{Participants}

All participants were recruited from a cross-sectional evaluation previously described elsewhere. ${ }^{9}$ In this longitudinal study we included 21 of the original 26 patients ( 3 died and 2 were lost to follow-up) and, 15 of the 17 initial healthy controls ( 1 died, and 1 was lost to follow-up). The healthy controls were recruited among hospital staff and patient's family members, using as exclusion criteria, any diagnosis of other skin disorder (e.g. psoriasis), connective tissue disease or rheumatic inflammatory disease. No significant differences were found between patients and controls, regarding age and gender.

All participants were submitted to a clinical and ultrasound evaluation at baseline and at follow-up, a median of $4.9(0.4)$ years later.

All SSc patients fulfilled the 2013 ACR/EULAR criteria for the classification of SSc. ${ }^{12}$ The disease was classified as diffuse cutaneous or limited cutaneous SSc, according to the extent of skin involvement. ${ }^{13}$

\section{Ethics}

Ethical approval was obtained from the Ethics Committee of Centro Hospitalar e Universitário de Coimbra (CHUC - 118-17). All patients and controls provided signed inform consent prior to any study procedures.

\section{Clinical skin thickness scoring (mRSS)}

Skin thickness was clinically assessed using the mRSS, scoring the palpation at each of 17 skin sites on a 0-3 scale. ${ }^{14}$ The same experienced rheumatologist (MJS) performed the mRSS at baseline and followup, on the same day of the skin ultrasound.

\section{Clinical phase of skin involvement}


Skin involvement phase was clinically assessed and classified as oedematous, fibrotic or atrophic by the same rheumatologist (MJS) at baseline and follow-up, on the same day of the skin ultrasound, following currently accepted descriptions. ${ }^{15-18}$

\section{Skin ultrasound evaluation}

Skin stiffness was measured at baseline and follow-up, through shear-wave elastography, using virtual touch image quantification (VTIQ), at the same 17 sites of the mRSS. SWE was performed with an ACUSON Ultrasound System (Siemens Healthcare), using a linear 4-9 MHz transducer. The ultrasound protocol has been described elsewhere. ${ }^{7}$ In brief, acceptance of an ultrasound image for analysis was based on clear visualization of an interface between the epidermis, dermis and subcutaneous tissues and on an automated image quality indicator provided by the ultrasound system. The sonographer placed sampling gates with the minimum possible size $(2 \times 2 \mathrm{~mm})$, over the dermis. The VTIQ output simultaneously displays a color-coded tissue stiffness map and absolute shear-wave velocity values (in $\mathrm{m} / \mathrm{s}$, up to $10 \mathrm{~m} / \mathrm{s}$ ) in one single image. Higher shear-wave velocities indicate greater tissue stiffness. The SWV for each site scanned was established as the mean of three consecutive measurements.

The same rheumatologist (TS) performed all ultrasound measurements, blinded for the attributed Rodnan skin score. The intra-observer reproducibility of SWE in this examiner's hands is reflected by intraclass correlation coefficients ranging from 0.70 (foot) to 0.98 (finger) in SSc; and 0.81 (thigh) to 0.97 (finger) in healthy controls (Table S1).

\section{Statistical methods}

Continuous variables were reported as means (standard deviation), if normally distributed or, median (interquartile range) if not normally distributed. Categorical variables were presented as frequencies. Differences between groups were analysed using the related-samples Wilcoxon Signed Rank test and the Mann-Whitney U test.

\section{Results}

\section{Clinical features}

Baseline clinical features of the patients with SSc and healthy controls are presented in table 1. All patients in an oedematous phase at baseline progressed to a fibrotic $(n=3)$ or atrophic phase $(n=2)$. Of the 16 patients in a fibrotic phase at baseline, 11 maintained the fibrotic phase and 5 progressed to an atrophic phase. 


\section{Changes in skin stiffness during follow-up}

Significant decreases in SWV values were observed in all Rodnan skin sites over the follow-up period ( $p \leq 0.001)$, except in the fingers (Table S2). mRSS only identified significant changes in the upper arm $(p=0.046)$ and forearm $(p=0.024)$ (Table S2).

Similar significant decreases in SWV values were observed in healthy controls in all skin sites $(p=0.001)$, except the leg.

At the second examination, SWVs in SSc patients became similar to that of controls in all sites, excepted the hands and fingers $(p=0.001)$ (Table $S 2)$.

The median percentage change in skin stiffness (i.e., \% change of SWV from baseline) was more pronounced in SSc than in controls. This difference reached statistical significance in the upper arm (median \% change $-53.2 \%$ in SSc vs $-41.5 \%$ in controls, $p=0.007$ ) (Figure 1 and Table 2 ). In addition, the \% change of SWV was variable in different skin sites (Table 2).

Taking all cases into account, and based in simple arithmetic, the effects of ageing seem to explain from $40 \%$ (leg) to $90 \%$ (chest) of the skin stiffness reduction observed in SSc patients. The only exception is the skin of the fingers, where disease is associated with a smaller decrease in skin stiffness than observed in healthy controls (data not shown).

\section{Skin stiffness and its progression according to the clinical phase of the disease}

Patients in an oedematous phase had higher SWV compared to patients in a fibrotic phase. These differences were statistically significantly at the abdomen, upper arm, forearm, hand and foot $(p<0.05)$.

The percentage change differed according to the phase of the disease at baseline (Table S3 and Supplementary Figure 2). Namely, patients in an oedematous phase at baseline had a higher percentage reduction in skin stiffness, in the majority of skin Rodnan sites, than patients in a fibrotic phase.

\section{Changes in skin stiffness according to form of the disease}

At baseline and follow-up, patients with a diffuse form had higher SWV values than patients with a limited form (Table S6). These differences were statistically significant at the upper arm, hand and finger $(p<0.05)$. However, there were no statistically significant differences in percentage change reduction in skin stiffness between patients with the limited and diffuse forms of SSc. 
The observation of higher SWV values compared with controls in sites with clinically unaffected skin $\left(\mathrm{mRSS}=0\right.$ ) at baseline, made in our original study was confirmed in this subgroup. ${ }^{7}$ (Table S4 and Table S5)

The longitudinal analyses demonstrated that SWV values also decreased significantly over the 5 years follow-up in all skin sites with Rodnan $=0$ at baseline (excepted in the fingers). There were no statistically significant differences between patients and controls at the end of follow-up in any of these sites (Table S4). Naturally, the Rodnan skin score could not identify any changes in such sites.

\section{Discussion}

This is the first study evaluating changes of skin stiffness over-time in patients with SSc, using shearwave elastography. This study provides evidence suggesting that skin stiffness (i.e. SWV values) decreased significantly in almost all Rodnan skin sites in SSc patients, as well as in healthy controls, over 5 years of follow-up. Shear-wave elastography was remarkably more sensitive to change over time than mRSS.

The observed decrease in stiffness follows the classical clinical expectation that skin in SSc evolves from an early oedematous status towards a fibrotic and finally an atrophic phase after reaching a maximal induration. ${ }^{15,16}$ In fact, at baseline, the five patients in oedematous phase had higher SWV values than patients in a fibrotic phase in the corresponding skin sites. During follow-up, SWV values decreased in almost all skin sites, which parallels the decline of oedema, the onset of fibrosis and, finally, atrophy.

Surprisingly, however, our observations in healthy controls suggest that a substantial part of the decrease in skin stiffness observed in patients with SSc is probably explained by normal skin ageing. Collagen fiber network of the dermis layer is known to change with aging and this is expected to affect the elasticity of this layer. ${ }^{18}$ In fact, Shuster et al measured the skin collagen and dermal thickness in skin biopsies obtained from the forearm of $\sim 150$ healthy controls. ${ }^{18}$ They demonstrated that skin collagen decreased with age, namely after the age of 20 in males and 50 in females. ${ }^{18}$ Another study by Leveque et al, found that skin thickness starts to decrease from the age of 45 years both in male and female, with female's skin becoming thinner that that of males. ${ }^{19}$ Interestingly, these findings were recently corroborated by a study using SWE to determine age-related changes of the skin in healthy controls. ${ }^{20}$ These authors demonstrated that SWV values decrease significantly in healthy controls older than 50 years compared with the 20- to 50-year group, at the finger and forearm. ${ }^{20}$ As the previous mentioned studies were cross-sectional, we cannot infer skin changes overtime from them. ${ }^{18-20}$ of note, in the present study $72.2 \%$ of the participants were older than 50 at baseline [60.3 (7.7) years]. Other factors, besides age itself, such as skin site, gender, hormonal phase and contextual factors may have also contributed to the observed changes and deserve consideration in future studies.

Our results suggest that elastography may be useful as an aid in distinguishing between changes in skin due to oedema and induration or sclerosis, a recognized limitation of mRSS. ${ }^{15}$ This may be particularly 
important in the assessment of the early phases of disease and response to treatment. Similar observations have been made in two longitudinal studies of ultrasound dermal thickness: thickness decreased and patients became more similar to the control population, between the $1^{\text {st }}$ and the $4^{\text {th }}$ years of follow-up. ${ }^{4,21}$ Kaloudi et al. found that dermal thickness decreased as the clinical phase progressed from the edematous to the atrophic phase. ${ }^{6}$

A relevant key message from our findings provide is the evidence that skin SWV evaluation is a more sensitive instrument to measure skin change over time than mRSS. In fact, SWE identified significant changes overtime at all skin sites (except fingers), where mRSS only showed significative differences in upper arm and forearm.

Another key message is the fact that SWE captured significant changes over time in skin sites with local normal mRSS at baseline. This is reinforced by the obvious fact that mRSS would, by definition, be unable to identify age-related skin changes in normal skin, and thus the impact of ageing in SSc.

These comparisons should, however, be interpreted in light of evidence that the mRSS and SWE measure different skin properties: mRSS measures not only thickness, but also texture and fixation, ${ }^{15}$ while elastography measures only skin stiffness. In future studies, it would be of interest not only to validate SWE against dermal thickness ultrasound or optical coherence tomography, but also against histologic findings.

We also observed that percentage SWV reduction was more pronounced in certain sites (chest, upper arm, and forearms) than in others. This is in line with studies that have identified the chest and forearms as the sites with more pronounced skin changes overtime, as opposed to the lower extremities, abdomen, fingers and face, which tend to be more stable. ${ }^{22}$ These findings raise the hypothesis that excluding relatively static skin sites may improve the sensitivity to change of total skin scores.

This is the first study addressing the sensitivity over time of SWE in SSc and controls. The same observers performed ultrasound evaluations and mRSS at baseline and follow-up. Although our data further supports the use of SWE as a potential outcome measure of skin involvement in SSc, its interpretation is limited by the small sample size, forcing a more descriptive than statistical subgroup analysis. Future skin ultrasound studies would benefit from a cohort of early diffuse patients with a shorter evaluation interval to further clarify whether changes are age- or disease-related, compared to later disease and healthy controls. It should also be considered that about half of the patients received immunosuppressive treatment between the two clinical and ultrasound evaluations: it cannot be ruled out that some of the changes observed were influenced by these medications.

\section{Conclusions}

In conclusion, findings reported herein highlight that a substantial part of the improvement of the skin in SSc may be explained by normal ageing. They support the higher discriminant ability of shear-wave 
elastography in detecting subtle skin changes not identified by mRSS. Further longitudinal studies with a higher number of patients in different phases of skin involvement are needed to fully clarify its potential. Establishing normal reference data for these ultrasound measurements may also foster earlier diagnosis.

\section{List Of Abbreviations}

CHUC: Centro Hospitalar e Universitário de Coimbra

mRSS: modified Rodnan skin score

SWE: shear wave elastography

SWV: shear-wave velocity

SSc: systemic sclerosis

VTIQ: Virtual Touch imaging and quantification

\section{Declarations}

Acknowledgments The authors would like to thank all the participants who contributed their time to this study.

Competing interests None declared.

Contributors TS, MJS, JAPS contributed to conception, design of the study and drafting of the manuscript; TS, MS contributed to the acquisition and analysis of the data. All authors contributed to revising the manuscript critically for important intellectual content.

Patient consent for publication Not required.

Ethics approval Centro Hospitalar e Universitário de Coimbra (CHUC - 118-17).

Funding The authors have not declared a specific grant for this research from any funding agency in the public, commercial or not-for-profit sectors. 
Data sharing statement All data relevant to the study are included in the article or uploaded as supplementary information.

Patient and Public involvement Statement Not required.

\section{References}

1. Denton CP, Khanna D. Systemic sclerosis. Lancet. 2017;390:1685 99.

2. Pope JE, Baron M, Bellamy N, Campbell J, et al. Variability of skin scores and clinical measurements in scleroderma. J Rheumatol. 1995;22:1271-6.

3. Kaldas M, Khanna PP, Furst DE, Clements PJ, et al; investigators of the human recombinant relaxin and oral bovine collagen clinical trials. Sensitivity to change of the modified Rodnan skin score in diffuse systemic sclerosis--assessment of individual body sites in two large randomized controlled trials. Rheumatology (Oxford). 2009;48:1143-6.

4. Hesselstrand R, Carlestam J, Wildt M, Sandqvist G, Andréasson K. High frequency ultrasound of skin involvement in systemic sclerosis - a follow-up study. Arthritis Res Ther. 2015;17:329.

5. Moore TL, Lunt M, McManus B, Anderson ME, Herrick AL. Seventeen-point dermal ultrasound scoring system-a reliable measure of skin thickness in patients with systemic sclerosis. Rheumatology (Oxford). 2003;42:1559-63.

6. Kaloudi O, Bandinelli F, Filippucci E, Conforti ML, Miniati I, Guiducci S, Porta F, Candelieri A, Conforti D, Grassiri G, Grassi W, Matucci-Cerinic M. High frequency ultrasound measurement of digital dermal thickness in systemic sclerosis. Ann Rheum Dis. 2010;69:1140-3. 
7. Santiago T, Alcacer-Pitarch B, Salvador MJ, Del Galdo F, Redmond AC, da Silva JA. A preliminary study using virtual touch imaging and quantification for the assessment of skin stiffness in systemic sclerosis. Clin Exp Rheumatol. 2016;100:137-141.

8. Hou Y, Zhu QL, Liu H, Jiang YX, Wang L, Xu D, Li MT, Zeng XF, Zhang FC. A preliminary study of acoustic radiation force impulse quantification for the assessment of skin in diffuse cutaneous systemic sclerosis. J Rheumatol. 2015;42:449-55.

9. Liu H, Hou Y, Zhu QL, Xu D, Wang L, Li JC, Jiang YX, Wang Q, Li MT, Zhang FC, Zeng XF. A preliminary study of skin ultrasound in diffuse cutaneous systemic sclerosis: Does skin echogenicity matter? PLoS One. 2017;12:e0174481.

10. Iagnocco A, Kaloudi O, Perella C, Bandinelli F, Riccieri V, Vasile M, Porta F, Valesini G, Matucci-Cerinic M. Ultrasound elastography assessment of skin involvement in systemic sclerosis: lights and shadows. J Rheumatol. 2010;37:1688-91.

11. Taljanovic MS, Gimber LH, Becker GW, Latt LD, Klauser AS, Melville DM, Gao L, Witte RS, Shear-Wave Elastography: Basic Physics and Musculoskeletal Applications, Radiographics. 2017;37:855-870.

12. van den Hoogen F, Khanna D, Fransen J, et al. 2013 classification criteria for systemic sclerosis: an American college of rheumatology/European league against rheumatism collaborative initiative. Ann Rheum Dis. 2013; 72:1747-55.

13. LEROY EC, BLACK C, FLEISCHMAJER R et al.: Scleroderma (systemic sclerosis): classification, subsets and pathogenesis. J Rheumatol 1988;15: 202-5.

14. Rodnan GP, Lipinski E, Luksick J. Skin thickness and collagen content in progressive systemic sclerosis and localized scleroderma. Arthritis Rheum. 1979;22:130-40. 
15. Czirják L, Foeldvari I, Müller-Ladner U.; Skin involvement in systemic sclerosis. Rheumatology (Oxford). 2008;47 Suppl 5:v44-5.

16. Shah AA, Wigley FM. My approach to the treatment of scleroderma. Mayo Clin Proc. 2013;88:377-93.

17. Khanna D, Furst DE, Clements PJ, Allanore Y, et al. Standardization of the modified Rodnan skin score for use in clinical trials of systemic sclerosis. J Scleroderma Relat Disord. 2017;2:11-18.

18. Shuster S, Black MM, McVitie E.The influence of age and sex on skin thickness, skin collagen and density. Br J Dermatol. 1975;93:639-43.

19. Leveque JL, Corcuff P, de Rigal J, Agache P. In vivo studies of the evolution of physical properties of the human skin with age. Int J Dermatol. 1984;23:322-9.

20. Yang Y, Wang L, Yan F, Xiang X, Tang Y, Zhang L, Liu J, Qiu L. Determination of Normal Skin Elasticity by Using Real-time Shear Wave Elastography. J Ultrasound Med. 2018;37:2507-2516.

21. Akesson A, Hesselstrand R, Scheja A, Wildt M. Longitudinal development of skin involvement and reliability of high frequency ultrasound in systemic sclerosis. Ann Rheum Dis. 2004;63:791-6.

22. Silman A, Harrison M, Brennan P. Is it possible to reduce observer variability in skin score assessment of scleroderma? The ad hoc International Group on the Assessment of Disease Outcome in Scleroderma. J Rheumatol. 1995;22:1277-80.

\section{Tables}

Table 1 - Clinical and demographic characteristics of the participants at baseline. Values are in median (Q1-Q3), unless stated otherwise.

RP: Raynaud phenomenon; ANA: Anti-nuclear antibody; ACA: Anti-centromere antibody; mRSS: modified Rodnan skin score.

aMethotrexate (average dose 15mg/week, N=2); Prednisolone or equivalent (average dose 


\begin{tabular}{|c|c|c|}
\hline & $\begin{array}{l}\text { SSc patients } \\
\quad(\mathrm{N}=21)\end{array}$ & $\begin{array}{l}\text { Controls } \\
(\mathrm{N}=15)\end{array}$ \\
\hline Female, n (\%) & 18 (85.7) & $11(73.3)$ \\
\hline Age (years) & $58.0(48.5-63.0)$ & $55.0(45.0-63.0)$ \\
\hline \multicolumn{3}{|l|}{ Smoking habits, n (\%) } \\
\hline Never & $17(80.9)$ & $11(73.3)$ \\
\hline Ex-smoker & $4(19.1)$ & $4(26.6)$ \\
\hline Disease duration since diagnosis, years & $10.0(5.5-14.0)$ & - \\
\hline Disease duration since $\mathrm{RP}$, years & $14.0(6.5-16.5)$ & - \\
\hline Limited form, $\mathrm{n}(\%)$ & $12(57.1)$ & - \\
\hline mRSS total & $8.0(4.0-15.0)$ & - \\
\hline Phase, n (\%) & & - \\
\hline Oedematous & $5(23.8)$ & \\
\hline Fibrotic & $16(76.2)$ & \\
\hline ANA positive, $\mathrm{n}(\%)$ & $20(95.2)$ & - \\
\hline ACA positive, n (\%) & $9(42.9)$ & - \\
\hline Anti-Scl 70 positive, $\mathrm{n}(\%)$ & $7(33.3)$ & - \\
\hline 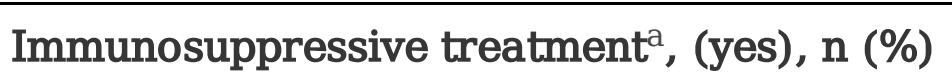 & $6 / 21$ & - \\
\hline Vasodilators treatment ${ }^{\mathrm{b}}$, (yes), n (\%) & $9 / 21$ & - \\
\hline
\end{tabular}

$5 \mathrm{mg} /$ day $(\mathrm{N}=4)$.

${ }^{b}$ Nifedipine (average dose 30mg/day, $\mathrm{N}=7$ ) and/or Pentoxifylline (average dose $800 \mathrm{mg} /$ day, $\mathrm{N}=5$ ).

Table 2 - Comparison of percentage changes in shear-wave velocity values, in each Rodnan site of analysis, observed in SSc patients and controls.

\footnotetext{
${ }^{\#}$ Values are in medians (Q1-Q3).

${ }^{\dagger}$ Mann-Whitney U test. NS, Non-significant. Statistically significant results are in bold.
}

\section{Figures}




\begin{tabular}{|c|c|c|c|}
\hline Rodnan sites & $\begin{array}{c}\text { SSc patients } \\
(\mathrm{n}=21)^{\#} \\
\end{array}$ & $\begin{array}{l}\text { Controls } \\
(\mathrm{n}=15)^{\#}\end{array}$ & $\begin{array}{c}\text { SSc vs controls } \\
{\text { ( } \mathrm{p} \text { value })^{\dagger}}\end{array}$ \\
\hline Chest & $-51.5 \%(--55.2$ to -41.7$)$ & $-46.9 \%(-50.0$ to -32.0$)$ & NS \\
\hline Abdomen & $-38.9 \%(-58.1$ to -28.7$)$ & $-32.6 \%(-43.9$ to -8.2$)$ & NS \\
\hline Upper arm & $-53.2 \%(-60.2$ to -40.7$)$ & $-41.5 \%(-48.7$ to -38.8$)$ & 0.007 \\
\hline Forearm & $-44.3 \%(-57.5$ to -40.7$)$ & $-37.1 \%(-52.0$ to -23.3$)$ & NS \\
\hline Hand & $-33.5 \%(-52.6$ to -19.9$)$ & $-26.2 \%(-38.6$ to -8.5$)$ & NS \\
\hline Finger & $-5.3 \%(-36.9$ to 34.7$)$ & $-24.6 \%(-29.8$ to -12.4$)$ & NS \\
\hline Thigh & $-37.1 \%(-45.3$ to -31.2$)$ & $-31.6 \%(-37.3$ to -24.0$)$ & NS \\
\hline Leg & $-25.8 \%(-37.8$ to -3.2$)$ & $-10.3 \%(-27.3$ to -6.9$)$ & NS \\
\hline Foot & $-36.4 \%(-56.6$ to -29.7$)$ & $-27.1 \%(-45.2$ to -14.3$)$ & NS \\
\hline
\end{tabular}

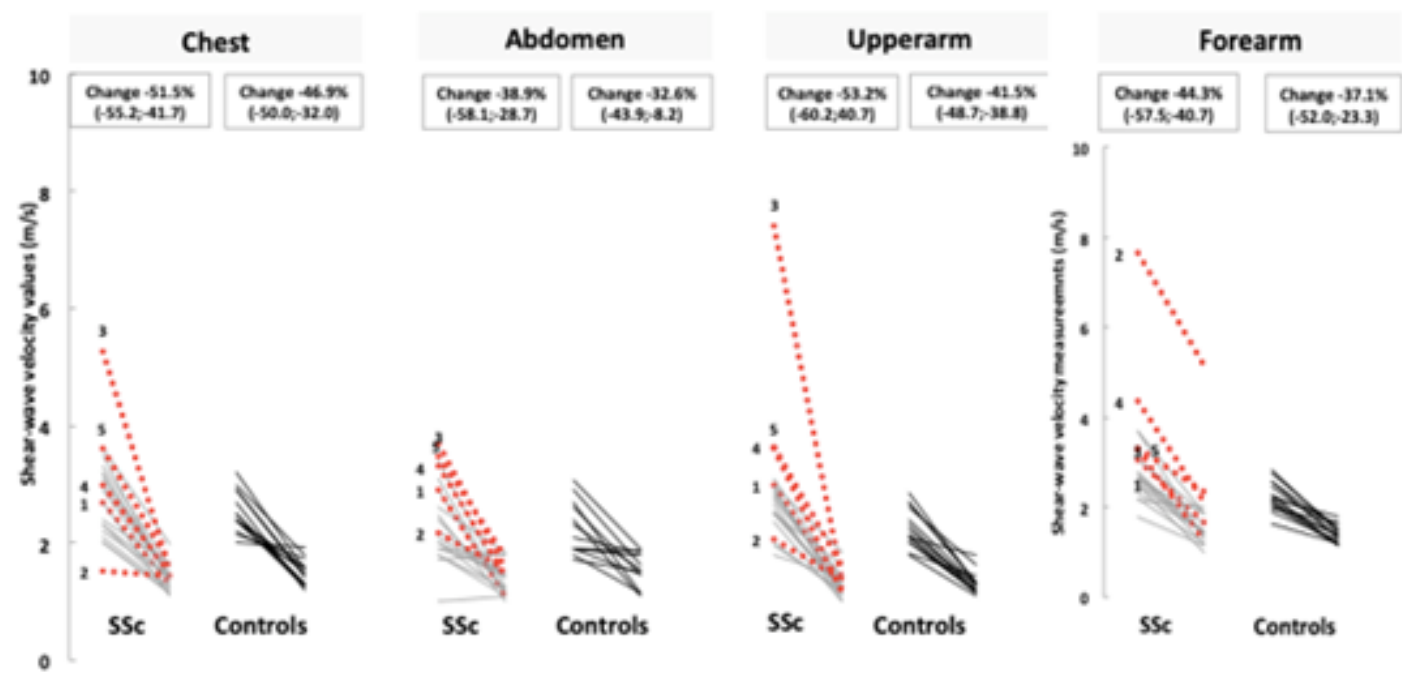




\section{Figure 1}

Shear-wave velocity values (m/s), measured by shear-wave elastography, at the Rodnan skin sites, at baseline and follow-up, in SSc and controls. Percentage change values are presented as median (Q1-Q3). Patients 2 and 3 progressed from oedematous to atrophic phase. Red dotted lines represent oedematous patients at baseline; and, the grey lines represent patients in fibrotic phase at baseline.

\section{Supplementary Files}

This is a list of supplementary files associated with this preprint. Click to download.

- SUPPLEMENTARY200207.doc 